\title{
Performance of a Tracking Device Based on the GEM Scintillation
}

\author{
F. A. F. Fraga, L. M. S. Margato, S. T. G. Fetal, R. Ferreira Marques, and A. J. P. L. Policarpo
}

\begin{abstract}
A tracking device based on gas electron multiplier scintillation using a high-resolution cooled charge-coupled device (CCD) camera was developed. The CCD is sensitive to the 400-1000 $\mathrm{nm}$ band, and standard glass photographic lenses are used. The active gas is an $\mathrm{Ar}-\mathrm{CF}_{4}$ mixture. The prototype detector is described and images of single alpha tracks are presented. The light yield and detector performance for several other gaseous mixtures, such as ${ }^{4} \mathrm{He}-\mathrm{CF}_{4}$, are also investigated.
\end{abstract}

Index Terms-Alpha particle, gas electron multiplier, tracking charge-coupled device (CCD).

\section{INTRODUCTION}

$\mathbf{T}$ HE USE of gaseous tracking chambers for trajectory imaging is well established in scientific fields such as radiochemistry, radiobiology, and microdosimetry. For this purpose the optical readout of gaseous avalanche detectors with charge-coupled device (CCD) cameras has been done in the past, but associated with VUV or UV emitting mixtures, expensive lenses, or optical systems and photocathodes with image intensifiers [1], [2].

In a recent work [3], we considered the possibility of using the gas electron multiplier (GEM) as an active scintillator for X-ray detection, using visible light optics and a standard high-resolution CCD camera for the readout. A two-dimensional large-area low-granularity gaseous position-sensitive detector for X-rays was developed. The results obtained with a $10 \times 10 \mathrm{~cm}^{2}$ GEM foil demonstrated that the position resolution with $8-\mathrm{keV} \mathrm{X}$-rays using a $\mathrm{Xe}-\mathrm{CO}_{2}$ mixture is better than $100 \mu \mathrm{m}(\mathrm{rms})$ and that operation is possible at count rates up to $10^{5} \mathrm{~mm}^{-2} \mathrm{~s}^{-1}$. To increase the sensitivity of the detector, two stacked GEMs were also used, resulting in an improved gain and higher light emission per detected photon.

Our previous work on the scintillation in the 400-1000 nm band has shown that the very high electric field in the GEM channels, needed for electron multiplication, is not the optimum electric field for light emission [4]. Although careful optimization of the two GEMs' operation improved its sensitivity, an enlarged collecting gap at the end of the detector improved the light emission by an order of magnitude, with a small deterioration in the spatial resolution. This improvement of the detector sensitivity is enough for recording single small energy deposits and made possible track imaging using a standard CCD camera.

Manuscript received November 5, 2000. This work was supported in part by the Portuguese FCT under Contract CERN/P/FIS/15199/99.

The authors are with the Laboratório de Instrumentação e Física Experimental de Partículas, LIP-Coimbra, and the Physics Department, University of Coimbra, 3004-516 Coimbra, Portugal (e-mail: francisco@lipc.fis.uc.pt).

Publisher Item Identifier S 0018-9499(02)01836-1.
Recently, after some research was done in scintillation properties, a gaseous mixture yielding high luminosity $\left(\mathrm{Ar}-\mathrm{CF}_{4}\right)$ was found [5]. In this paper, we describe a gaseous tracking detector that we built using a standard CCD and glass optical system operating in the $400-1000 \mathrm{~nm}$ band and its performance.

\section{The Gain And Scintillation of $\mathrm{Ar}-\mathrm{CF}_{4}$}

Our first studies of the scintillation of avalanches in microstructures were done with $\mathrm{Ar}-\mathrm{CO}_{2}$ mixtures.

Although it was found that the addition of $\mathrm{CO}_{2}$ to Ar decreased the light emission, the amount of emitted light in $\mathrm{Ar}$ has a strong dependence on the purity of the gas. A few percent of quencher should be added to stabilize its operation [6].

Gas mixtures based on $\mathrm{CF}_{4}$ have been widely used in detectors, mainly due to its high drift velocity and optical and scintillation properties. Although the most studied photon emissions are in the far $\mathrm{UV}, \mathrm{CF}_{4}$ also emits visible and near-infrared photons [7]. Replacing the $\mathrm{CO}_{2}$ with $\mathrm{CF}_{4}$ should result in a detector with good counting and localization properties but higher scintillation yields. It is known that $\mathrm{CF}_{4}$ is transparent to its own photons, resulting in maximum gain limitations due to photon feedback in wire detectors, but the GEM geometry is intrinsically free from photon feedback [8]. Cascading GEMs even improves this shielding effect.

To study the operation of the GEM with $\mathrm{Ar}-\mathrm{CF}_{4}$ mixtures, we made measurements of charge and scintillation using our setup.

As the experimental system and detector that were used to obtain these results are similar to those described in [3], we will only refer to the more relevant aspects. The entrance window of the test chamber was made from $12.5-\mu \mathrm{m}$ aluminum foil, and a $10 \times 10 \mathrm{~cm}^{2}$ GEM foil was used for the measurements. The GEM and the drift grids were supported by fiberglass frames. The GEM, supplied by the CERN Gas Detector Development Group, was manufactured from a 50- $\mu \mathrm{m}$-thick copper coated kapton foil by a chemical etching process that produces holes with a double conical shape (standard shape) [9]. The diameters of the metal and kapton holes were 80 and $50 \mu \mathrm{m}$, respectively. The pitch was $140 \mu \mathrm{m}$ and the optical transparency $\approx 12 \%$. The GEM front electrode was grounded and the back one was operated at negative voltage. A drift plane was placed $4 \mathrm{~mm}$ before the GEM. No collecting electrode was used on these experiments. The avalanche electrons were collected by the GEM outer electrode. The light window, placed $5 \mathrm{~mm}$ behind the GEM, was made with a 75- $\mu \mathrm{m}$ mylar foil.

The ratio between the secondary and the primary currents, measured at the GEM back electrode and operating the chamber in ionization mode, respectively, was used to calculate the GEM 


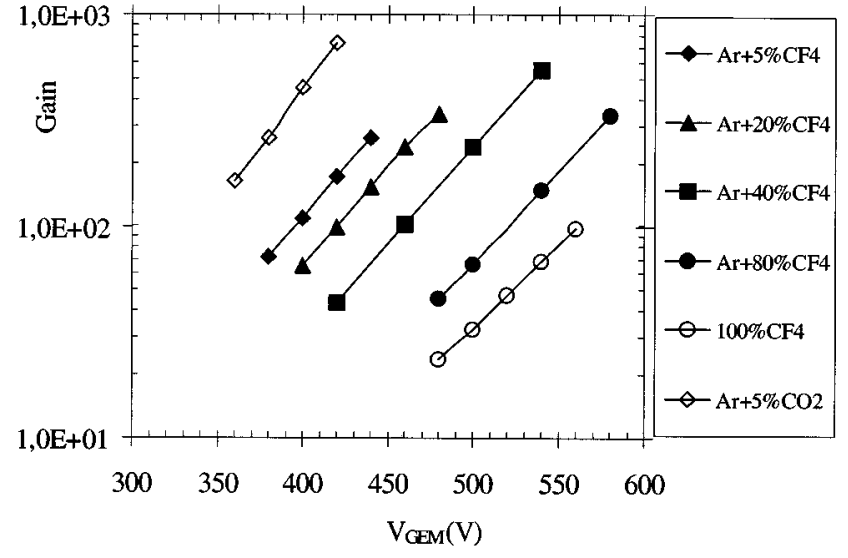

Fig. 1. GEM gains measured in $\mathrm{Ar}-\mathrm{CF}_{4}$ as a function of $V_{\mathrm{GEM}}$. $\mathrm{Ar}-5 \% \mathrm{CO}_{2}$ values are shown for comparison.

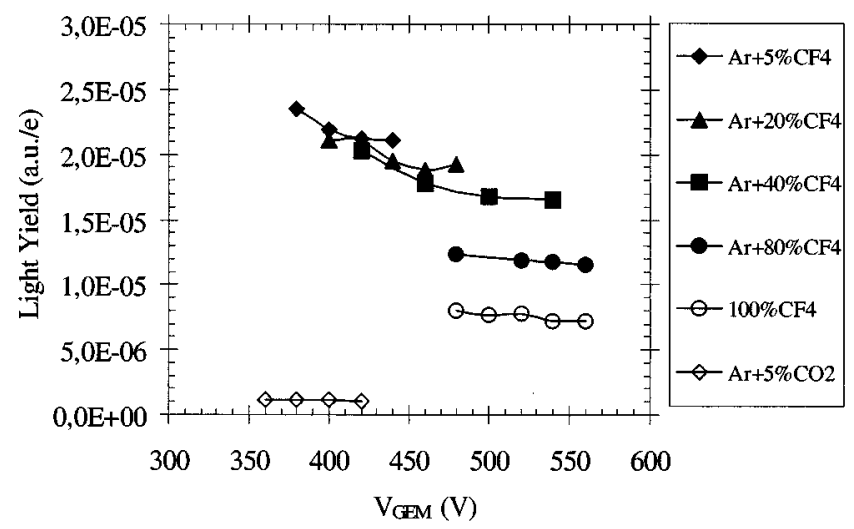

Fig. 2. Ratio of emitted light over secondary electron current versus $V_{\text {GEM }}$ for several quencher concentrations of the $\mathrm{Ar}-\mathrm{CF}_{4}$ mixture. $\mathrm{Ar}-5 \% \mathrm{CO}_{2}$ values are shown for comparison.

gains. A Quantix 1400 camera, manufactured by Photometrics Ltd., was used to read out the light emitted from the GEM. It uses a Peltier-cooled low-noise-CCD KAF 1400 from Kodak, with $1317 \times 1035$ pixels of $6.8 \times 6.8 \mu \mathrm{m}^{2}$. The spectral response goes from 400 to $1000 \mathrm{~nm}$. All the pictures presented in this paper were taken with the CCD cooled to $-30{ }^{\circ} \mathrm{C}$. A standard 50-mm f: 1.8 photographic lens was used. The camera was placed at the minimum allowable focusing distance, about $30 \mathrm{~cm}$ away from the chamber mylar window.

Fig. 1 shows the gain-voltage characteristic of the GEM in $\mathrm{Ar}-\mathrm{CF}_{4}$ for several quencher concentrations. As expected, GEMs have to be operated with higher $V_{\mathrm{GEM}}$ when using $\mathrm{CF}_{4}$, and the maximum gain is smaller than with $\mathrm{CO}_{2}$ mixtures.

The variation of the detected light divided by the secondary current versus $V_{\mathrm{GEM}}$ for several quencher concentrations of the $\mathrm{Ar}-\mathrm{CF}_{4}$ gas filling is shown in Fig. 2. This figure, in arbitrary units, is a good parameter to compare relative scintillation yields of gas mixtures if the geometry of the system is kept unchanged. The data on $\mathrm{Ar}-5 \% \mathrm{CO}_{2}$ are shown for comparison. $\mathrm{Ar}-5 \% \mathrm{CF}_{4}$ has a much greater light emission than $\mathrm{Ar}-5 \% \mathrm{CO}_{2}$, almost 20 times larger, and considerable light emission for higher percentage of quencher, contrary to what was observed in $\mathrm{Ar}-\mathrm{CO}_{2}$.

The very high scintillation yield of $\mathrm{Ar}-5 \% \mathrm{CF}_{4}$ opens new areas of research in GEM imaging, resulting in detectors of much increased sensitivity.

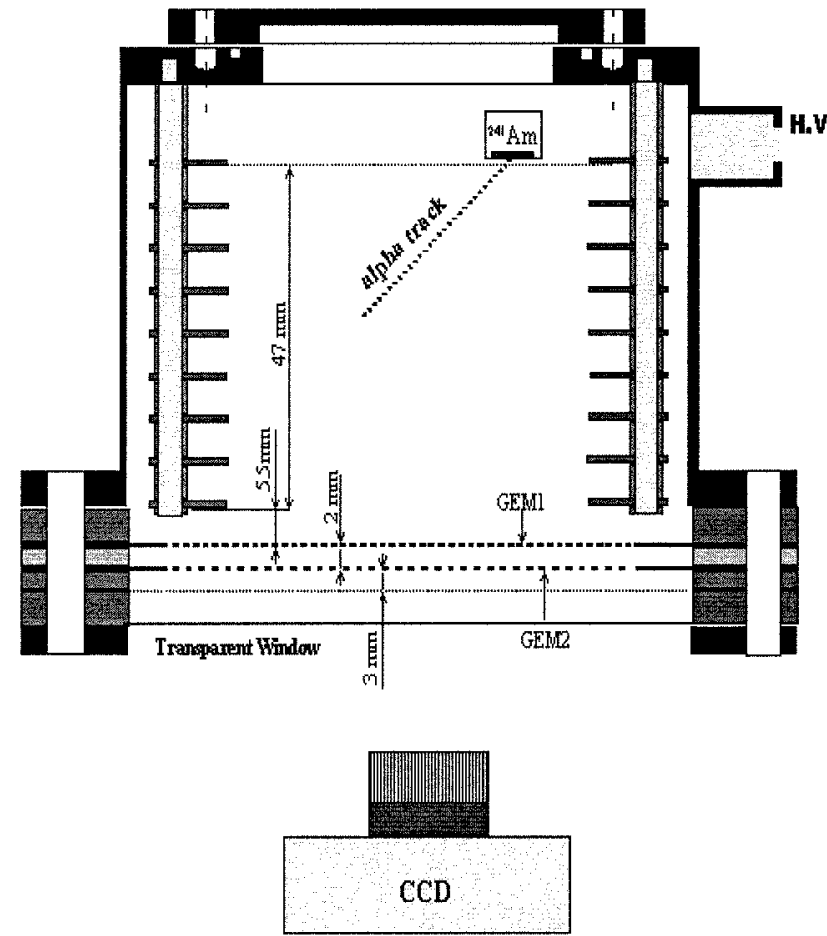

Fig. 3. Schematic cross-section of the tracking chamber used in this paper. The CCD (not drawn to scale) was placed $30 \mathrm{~cm}$ away from the mylar window.
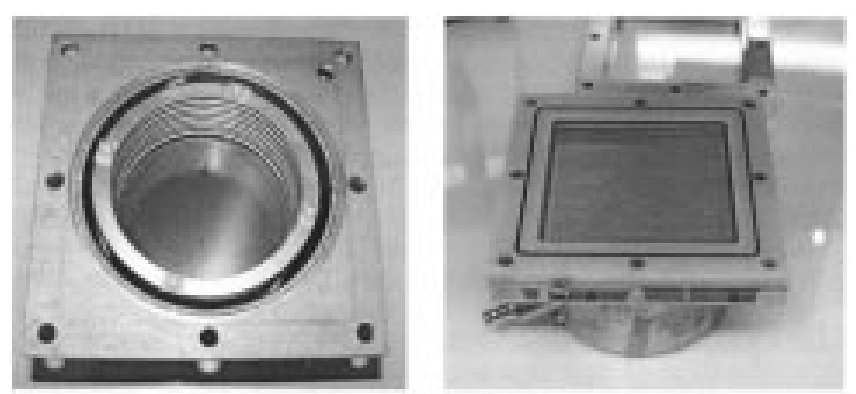

Fig. 4. Photographs of the tracking chamber drift zone and of the chamber with the mylar window removed, showing the outer GEM.
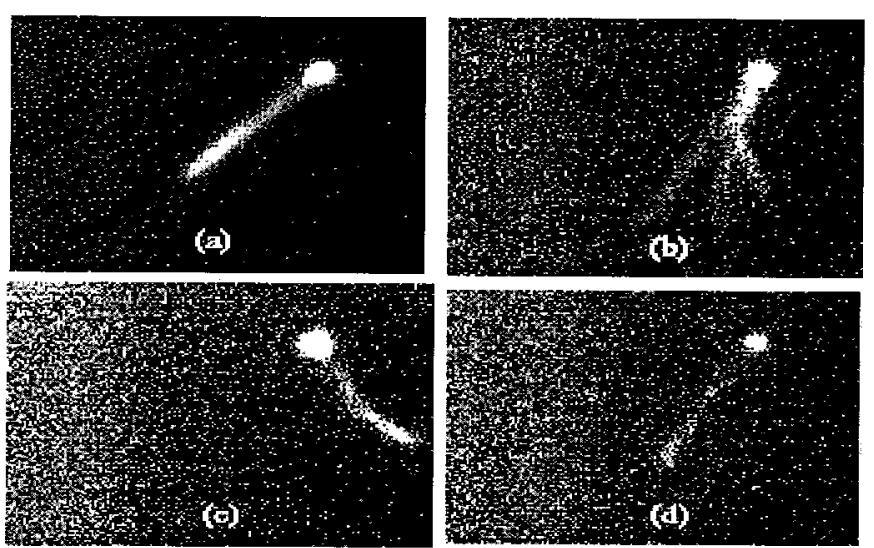

Fig. 5. Images of alpha tracks taken using the tracking chamber with $\mathrm{Ar}-5 \%$ $\mathrm{CF}_{4}$. (a), (b) $V_{\text {GEM1 }}=V_{\text {GEM } 2}=400 \mathrm{~V}$ (Gain $\left.\sim 140\right), E_{T}=5.45 \mathrm{KV} / \mathrm{cm}$, $E_{C}=5.86 \mathrm{KV} / \mathrm{cm}$, CCD Binning $4 \times 4, T \exp .=10 \mathrm{~ms}$. (c), (d) $V_{\text {GEM1 }}=$ $V_{\text {GEM2 }}=430 \mathrm{~V}($ Gain $\sim 300), E_{T}=5.45 \mathrm{KV} / \mathrm{cm}, E_{C}=0$, CCD Binning $7 \times 7, T=10 \mathrm{~ms}$. 

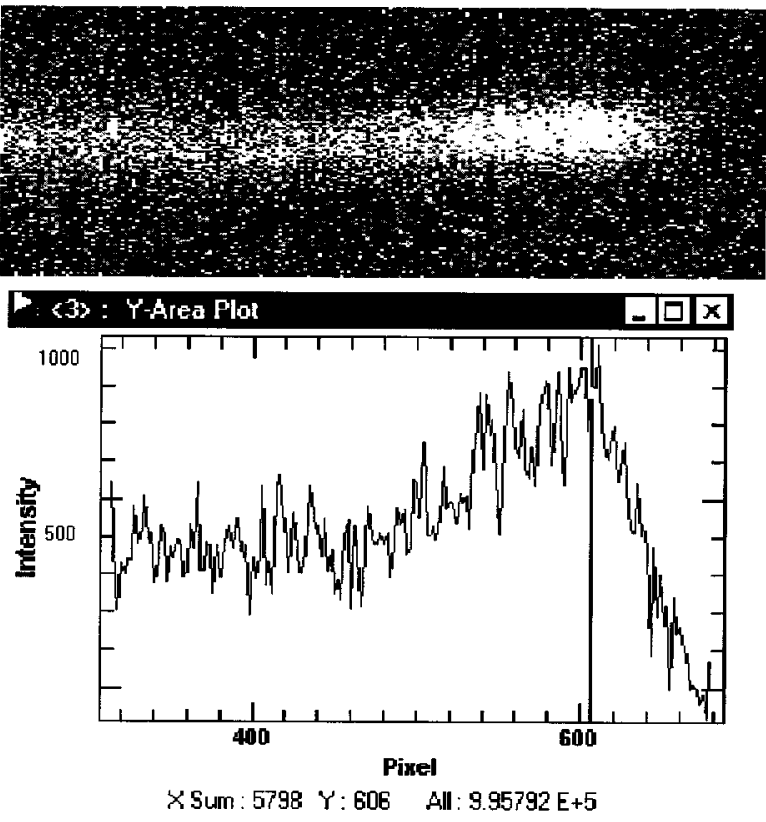

Fig. 6. Distribution of measured scintillation along an alpha track. The Bragg curve is revealed.

\section{DeVelopment of A Tracking Chamber}

We presented the possibility of using the double GEM chamber as a tracking detector in [10]. Since then, we built a tracking chamber with a sensitive volume of $\sim 250 \mathrm{~cm}^{3}$ that can image track lengths up to $8 \mathrm{~cm}$. The schematic drawing of the chamber is shown in Fig. 3. The chamber is fitted with two $10 \times 10 \mathrm{~cm}^{2}$ cascaded GEMs, separated by $2 \mathrm{~mm}$, and a collecting/multiplying electrode placed $3 \mathrm{~mm}$ away from the outer GEM. The GEM pitch was $140 \mu \mathrm{m}$, and the copper and kapton holes diameters were 80 and $60 \mu \mathrm{m}$. Two views of the chamber are presented in Fig. 4, showing the drift zone with the field rings and a detail of GEM assembly.

The chamber was filled with $\mathrm{Ar}-5 \% \mathrm{CF}_{4}$ and the $\mathrm{CCD}$ camera was again placed $30 \mathrm{~cm}$ from the chamber transparent mylar window. A low counting rate ${ }^{241} \mathrm{Am}$ alpha source was placed in the chamber, just over the drift electrode. The 5.48-MeV particles have a range of $4.5 \mathrm{~cm}$ in Ar.

In this paper, we will call $E_{D}, E_{T}$, and $E_{C}$, respectively, the drift field, the transfer field between GEMs, and the collection field between the outer GEM and the collecting electrode. When using $\mathrm{CF}_{4}$ mixtures, needing higher $V_{\mathrm{GEM}}$ voltage, the transfer field was found to be critical and was carefully optimized by simulation and experimental measurements.

The images of tracks obtained with several values of GEM gain and collection electric field are shown in Fig. 5. Images (a) and (b) were obtained with a collecting field of $5.9 \mathrm{kV} / \mathrm{cm}$, which increases gain and scintillation. Increasing the GEM gain and the CCD pixel binning, which improves the CCD signal-tonoise ratio, we could also operate the chamber without collection field, as seen in (c) and (d). From these data, we estimated that 200 photons were detected by the CCD per deposited $\mathrm{keV}$ in the gas.

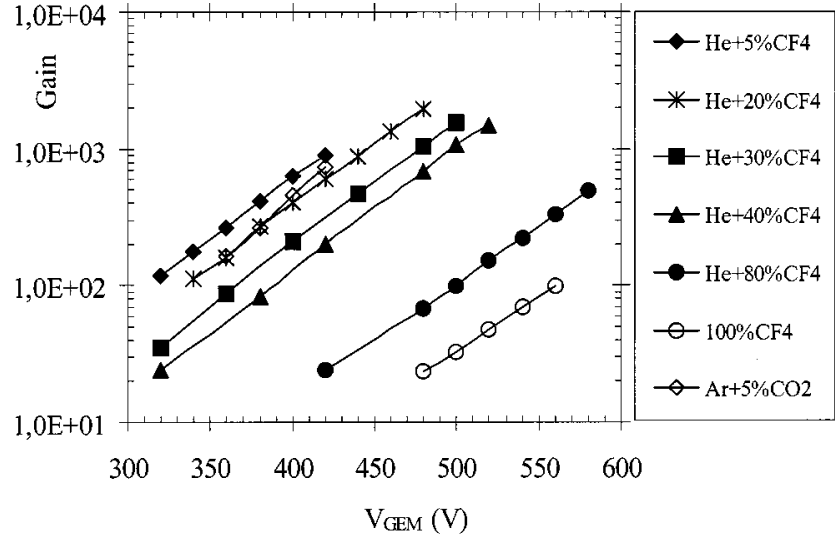

Fig. 7. GEM gains measured in $\mathrm{He}-\mathrm{CF}_{4}$ as a function of $V_{\mathrm{GEM}}$. $\mathrm{Ar}-5 \% \mathrm{CO}_{2}$ values are shown for comparison.

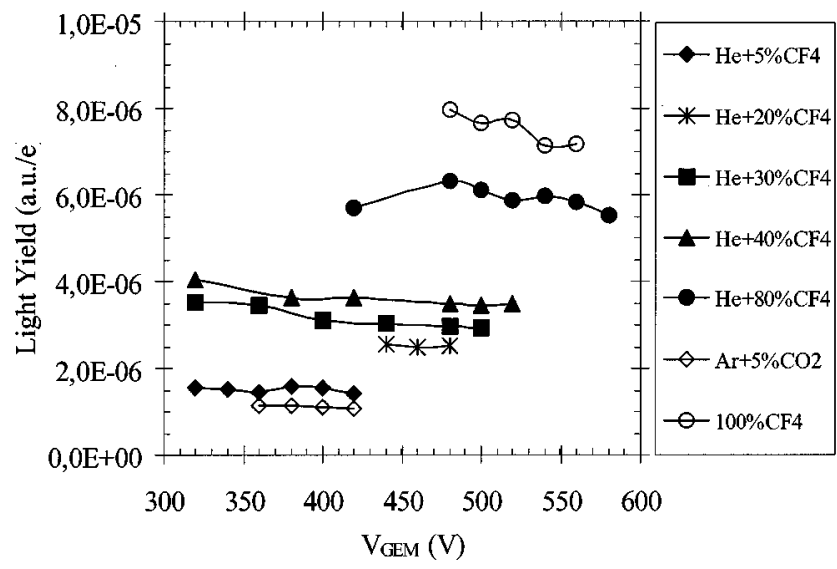

Fig. 8. Ratio of emitted light over secondary electron current versus $V_{\text {GEM }}$ for several quencher concentrations of the $\mathrm{He}-\mathrm{CF}_{4}$ mixture. $\mathrm{Ar}-5 \% \mathrm{CO}_{2}$ values are shown for comparison.

Fig. 6 shows in more detail an image of a single track and the distribution of the scintillation light along the longitudinal CCD pixels of the track. The familiar Bragg curve is revealed.

\section{IV. $\mathrm{He}-\mathrm{CF}_{4}$ RESULTS}

${ }^{3} \mathrm{He}-\mathrm{CF}_{4}$ is commonly used in gaseous neutron detectors [11]. The ${ }^{3} \mathrm{He}$ is used for neutron conversion $\left({ }^{3} \mathrm{He}+\mathrm{n} \Rightarrow \mathrm{p}+\right.$ $\left.{ }^{3} \mathrm{H}+770 \mathrm{keV}\right)$ and the $\mathrm{CF}_{4}$ is needed to decrease the range of the proton and the triton. Considering that the proton and the triton ranges in $\mathrm{CF}_{4}$ are 4.4 and $1.6 \mathrm{~mm}$, respectively, and that the mean energy deposit of alpha particles per millimeter in the preceding section $(\sim 120 \mathrm{keV} / \mathrm{mm})$ is similar to the energy density of the track resulting from the neutron absorption, we decided to perform some preliminary measurements in ${ }^{4} \mathrm{He}-\mathrm{CF}_{4}$ mixtures.

The experimental setup and operating mode were the same as in Section II. The gain as a function of the GEM voltage for different $\mathrm{CF}_{4}$ concentrations is shown in Fig. 7. The data for $\mathrm{Ar}-5 \% \mathrm{CO}_{2}$ are added for comparison. As already reported by several authors for other types of microstructures, the $\mathrm{He}$ mixtures have higher maximum gain before breakdown than the Ar mixtures. The variation of the light over secondary current ratio versus $V_{\mathrm{GEM}}$ for several quencher concentrations of the $\mathrm{He}-\mathrm{CF}_{4}$ gas filling is shown in Fig. 8. Despite the fact that the 
luminosity of $\mathrm{He}-\mathrm{CF}_{4}$ is lower than $\mathrm{Ar}-\mathrm{CF}_{4}$, it is still five times higher than $\mathrm{Ar}-\mathrm{CO}_{2}$.

These preliminary measurements show that integrating position detectors for neutrons could be developed using scintillation readouts.

\section{CONCLUSION}

Our findings that $\mathrm{Ar}-\mathrm{CF}_{4}$ mixtures have much higher luminosity than $\mathrm{Ar}-\mathrm{CO}_{2}$ made possible the development of an imaging chamber for single events using GEM scintillation read out by common CCD systems. The detector is currently being improved with an additional third GEM. Studies on the dependence of the scintillation versus pressure and GEM hole dimension have been started.

The optical readout of ${ }^{3} \mathrm{He}$ neutron detectors was shown to be feasible. We will soon perform tests in a neutron beam.

\section{ACKNOWLEDGMENT}

The authors would like to thank F. Sauli who made available the GEM foils used in this paper.

\section{REFERENCES}

[1] M. Susuky, "On the optical readout of gas avalanche chambers and its applications," Nucl. Instrum. Methods, vol. A263, pp. 237-242, 1988.

[2] U. Titt, "A Time projection chamber with optical readout for charged particle track stucture imaging," Nucl. Instrum. Methods, vol. A416, pp. 85-96, 1998.

[3] F. A. F. Fraga, S. T. G. Fetal, L. M. S. Margato, I. Ivaniouchenkov, R. Ferreira Marques, and A. J. P. L. Policarpo, "Imaging detectors based on the Gas Electron Multiplier scintillation light," presented at the Nuclear Science Symp. Medical Imaging Conf., Seattle, WA, Oct. 24-30, 1999.

[4] M. M. Fraga, S. T. G. Fetal, F. A. F. Fraga, E. Antunes, J. Gonçalves, C. C. Bueno, R. Ferreira Marques, and A. J. P. L. Policarpo, "Study of scintillation light from microstructure based detectors," IEEE Trans. Nucl. Sci., vol. 47, pp. 933-938, June 2000.

[5] M. M. Fraga, C. C. Bueno, J. A. C. Gonçalves, F. A. F. Fraga, E. Antunes, R. Ferreira Marques, and A. J. P. L. Policarpo, "Pressure dependence of secondary NIR scintillation in Ar and Ar/CF," IEEE Trans. Nucl. Sci., vol. 48, pp. 330-335, June 2001.

[6] F. A. F. Fraga, S. T. G. Fetal, R. Ferreira Marques, and A. J. P. L. Policarpo, "Quality control of GEM detectors using scintillation techniques," Nucl. Instrum. Methods, vol. A 442, pp. 417-422, 2000.

[7] Pansky, "The scintillation of $\mathrm{CF}_{4}$ and its relevance to detection science," Nucl. Instrum. Methods, vol. A364, p. 262, 1995.

[8] R. Chechik, "First results on the GEM operated at low gas pressures," Nucl. Instrum. Methods, vol. A4196, p. 423, 1998

[9] R. Bouclier, M. Capeáns, W. Domonik, M. Hoch, J.-C. Labbé, G. Million, L. Ropelewski, F. Sauli, and A. Sharma, "The Gas Electron Multiplier (GEM)," IEEE Trans. Nucl. Sci., vol. 44, pp. 646-650, June 1997.

[10] F. A. F. Fraga, L. M. S. Margato, S. T. G. Fetal, M. M. F. R. Fraga, R. Ferreira Marques, and A. J. P. L. Policarpo, "Optical readout of GEMS," Nucl. Instrum. Methods, submitted for publication.

[11] B. Guerard, "Neutron gas detectors at ILL," in Imaging 2000, Stockholm, Sweden, June 28-July 1, 2000. 